\title{
Experimental and numerical results of active flow control on a highly loaded stator cascade
}

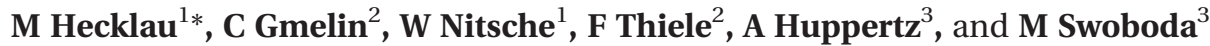 \\ ${ }^{1}$ Department of Aeronautics and Astronautics, Technische Universität Berlin, Berlin, Germany \\ ${ }^{2}$ Department of Fluid Mechanics and Engineering Acoustics, Technische Universität Berlin, Berlin, Germany \\ ${ }^{3}$ Rolls-Royce Deutschland Ltd \& Co. KG, Dahlewitz, Germany
}

The manuscript was received on 12 April 2011 and was accepted after revision for publication on 20 April 2011.

DOI: 10.1177/0957650911410156

\begin{abstract}
This article presents experimental and numerical results for a compressor cascade with active flow control. Steady and pulsed blowing has been used to control the secondary flow and separation characteristics of a highly loaded controlled diffusion airfoil. Investigations were performed at the design incidence for blowing ratios from approximately 0.7 to 3.0 (jet-to-inlet velocity) and a Reynolds number of 840000 (based on axial chord and inlet velocity). Detailed flow field data were collected using a five-hole pressure probe, pressure taps on the blade surfaces, and time-resolved Particle Image Velocimetry. Unsteady Reynolds-averaged Navier-Stokes simulations were performed for a wide range of flow control parameters. The experimental and numerical results are used to understand the interaction between the jet and the passage flow. The benefit of the flow control on the cascade performance is weighted against the costs of the actuation by introducing an efficiency which takes the presence of the jets into account.
\end{abstract}

Keywords: active flow control, compressor, stator cascade, URANS, PIV

\section{INTRODUCTION}

Flow control applications in turbomachinery have a great potential to break fundamental barriers [1]. With active flow control (AFC), the pressure ratio of one stage can be increased, and thus the same overall pressure ratio reached within a reduced number of stages. This leads to more compact and lighter jet engines, and thus decreases the manufacturing and operating costs of axial turbomachinery [2].

Active flow control methods with focus on rotor instabilities have been investigated by Bright et al. [3]. Passive and AFC applications focusing on boundary layer separation and secondary flow structures were tested on linear stator cascades by Hergt et al. [4]. Active, steady-state methods for controlling

*Corresponding author: Department of Aeronautics and Astronautics, Technische Universität Berlin, Marchstrasse 12, 10587 Berlin, Germany.

email: martin.hecklau@ilr.tu-berlin.de strong secondary flow structures as well as boundary layer separation were experimentally and numerically investigated by Nerger [5]. According to Greenblatt and Wygnanski [6], AFC by means of periodical addition of momentum is able to decrease the costs by an order of magnitude compared to steadystate blowing.

Within this work, a linear compressor cascade is investigated. Due to the critical loading, the low aspect ratio, and the high flow turning of the blades, a fully three-dimensional (3D) flow field with boundary layer separation is developing [7]. Figure 1 shows the complex flow topology within the passage with the help of experimental and numerical data. The corner vortex is represented by the decelerated areas on cross-planes along the blade. An oil flow visualization and wall-shear lines illustrate the $3 \mathrm{D}$ flow topology and separation. Numerically calculated streamlines near the walls give deeper insight into the $3 \mathrm{D}$ flow field. The general flow pattern observed is as follows: The laminar boundary layer separates from the blade suction side surface. The separated flow 

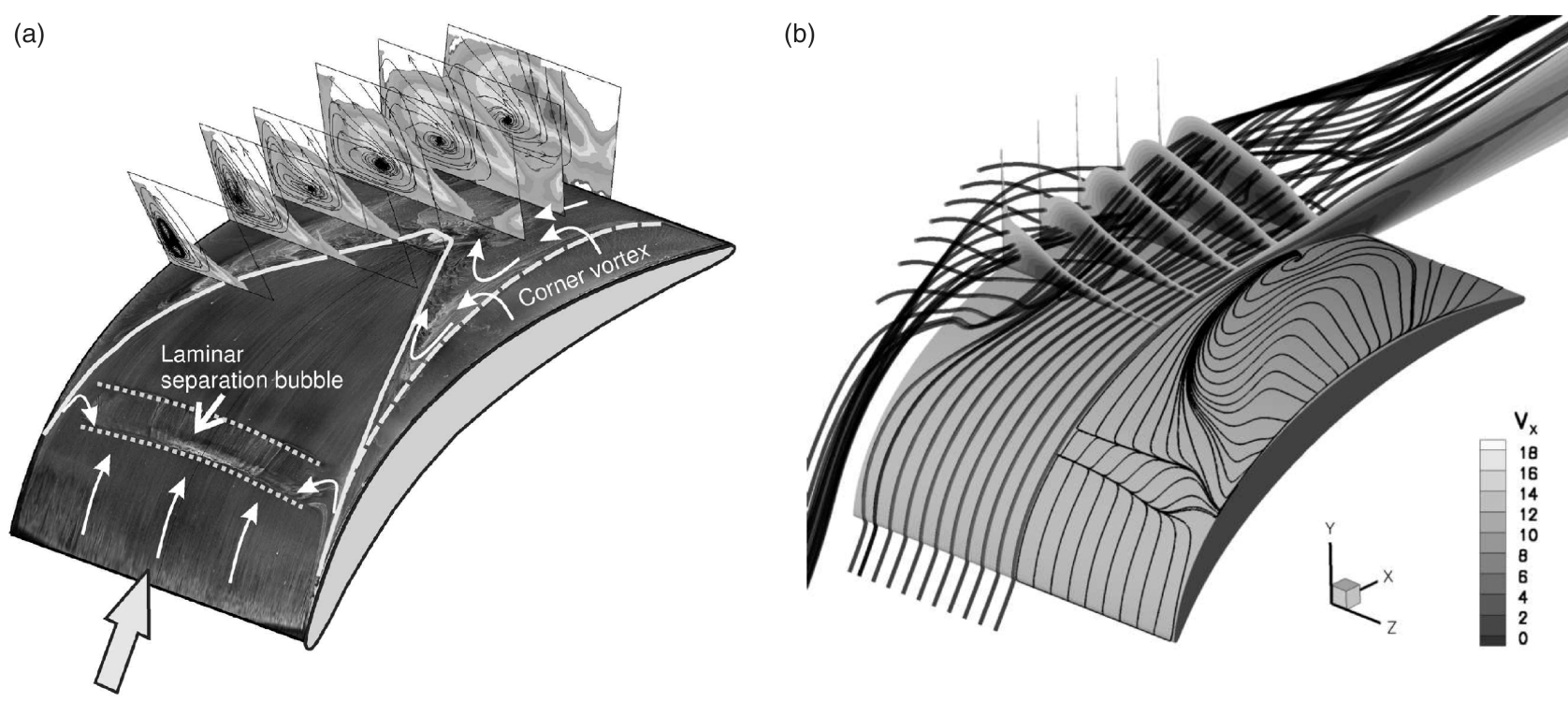

Fig. 1 Passage flow topology: (a) experimental measurement and (b) numerical simulation

undergoes transition and reattaches on the blade suction surface forming a laminar separation bubble. At the streamwise position of the turbulent reattachment, secondary flows come into effect. The main flow is narrowed in streamwise direction. Between the secondary and the main flows, a 3D separation line is formed ending up at midspan, where the flow separation occurs.

Two flow control concepts are adapted to the secondary flow pattern. Blowing out of the sidewalls reduces the blockage of the passage. Excitation of the flow by blowing out of the suction surface prevents the flow separation. The AFC approaches are investigated separately and in combination. Details on the time-resolved Particle Image Velocimetry methods can be found in Hecklau et al. [8]. In previous experimental and numerical works, a wide range of the parameters characterizing the pulsed excitation have been analysed [9-12].

In this article, the physics involved within the flow control are presented with the help of detailed flow field measurements. Comparisons with numerical results give an idea of the feasibility to predict the phenomena of flow control with respect to the known limitations and uncertainties involved in computational fluid dynamics (CFD). Previously performed evaluation of the global impact of the flow control by means of static pressure rise and total pressure loss of the cascade passage flow showed that correct trends can be predicted numerically. Within the scope of this article, the efficiency of the compressor cascade with and without actuation is considered.

\section{APPARATUS AND PROCEDURES}

Rolls-Royce Deutschland provided the cascade and blade geometry. The blades are designed with an overcritical turning of $\Delta \beta=60^{\circ}$ and a low aspect ratio of $A R=0.8$. Within the experiment, the cascade consists of seven blades. In order to obtain a sufficient thickness of the blade for integration of the AFC devices, the geometry of the cascade is scaled up to a blade height of $h=0.3 \mathrm{~m}$.

The according chord length is $l=0.375 \mathrm{~m}$. At the design point, the inflow Mach number is $M a_{1}=0.1$. With respect to the blade chord, the Reynolds number is $R e_{l}=840000$. An overview of the stator cascade is shown in Fig. 2 and the geometrical parameters are summarized in Table 1.

\subsection{Experimental setup and instrumentation}

The cascade test rig used for the experimental investigations is shown in Fig. 3. A homogeneous inlet flow is achieved by two additional tailboards and boundary layer suction at the end of the horizontal end walls. At each sidewall, 14 static pressure taps are installed for monitoring the inlet flow uniformity. The cascade test rig is operated in an open wind tunnel. Corresponding to the design Mach number of $M a_{1}=0.1$ and a constant total temperature of $T_{t, 1}=294 \mathrm{~K}$, the velocity at the inlet is $V_{1}=34 \mathrm{~m} / \mathrm{s}$. The wind tunnel turbulence intensity for $R e_{l}=840000$ is $T u=2.1$ per cent at the cascade inlet plane. The turbulence intensity is measured with a hot-wire probe and the signal is low-pass filtered with a cut-off frequency of $1 \mathrm{kHz}$. 


\subsubsection{Sidewall actuators - SW}

Steady and pulsed blowing out of slots integrated in the cascade sidewalls is used as an active method of controlling the dominant secondary flow structures. For this purpose, the air is blown through slots at a chord length of $x / l=10$ per cent, as can be seen in Figs 4(a) and (b). The slot's geometry is $20 \mathrm{~mm}$ in height and $0.4 \mathrm{~mm}$ in width. According to numerical investigations $[\mathbf{1 1}, \mathbf{1 3}]$, the injection angle in main

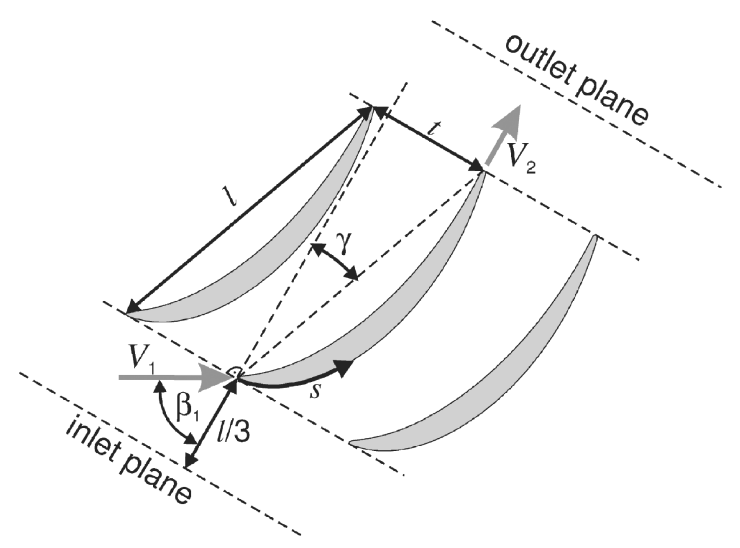

Fig. 2 Cascade geometry

Table 1 Cascade and blade geometry data

\begin{tabular}{llll}
\hline Parameter & Variable & Value & Unit \\
\hline Chord length & $l$ & 0.375 & $\mathrm{~m}$ \\
Blade pitch & $t$ & 0.15 & $\mathrm{~m}$ \\
Blade height & $h$ & 0.3 & $\mathrm{~m}$ \\
Stagger angle & $\gamma$ & 20 & $\mathrm{deg}$ \\
Turning & $\Delta \beta$ & 60 & $\mathrm{deg}$ \\
De Haller number & $\mathrm{DH}$ & 0.5 & 1 \\
\hline
\end{tabular}

(a)

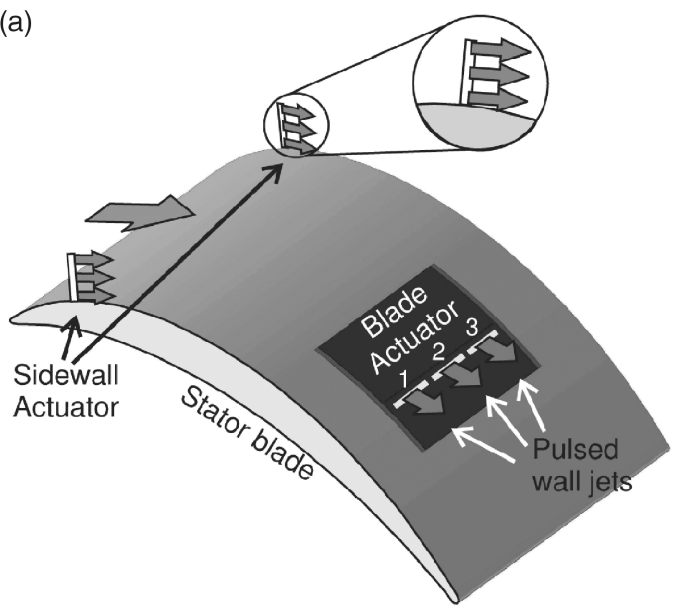

flow direction to the sidewall is set to $15^{\circ}$. In order to guarantee symmetrical flow conditions while actuating, all passages of the cascade are equipped with actuators at both sidewalls.

\subsubsection{Blade actuators - BA}

The flow separation on the suction surface of the blade is suppressed by actuators installed inside the stator blade. The actuator system consists of three slot-segments which can be addressed individually, cf. Fig. 4 (a), with the middle segment BA2 and the outer segments BA1 +3 . The slots are orientated in spanwise direction and have a geometry of $50 \mathrm{~mm}$ in length and $0.4 \mathrm{~mm}$ in width. The distance between the segments is kept as small as possible, resulting in a separation of $2 \mathrm{~mm}$.

The wall jet has a blowing angle of $45^{\circ}$ to the local blade surface, cf. Fig. 4 (c). Due to the shape of the actuator cavity, a uniform velocity distribution in spanwise direction can be reached. Fast-switching

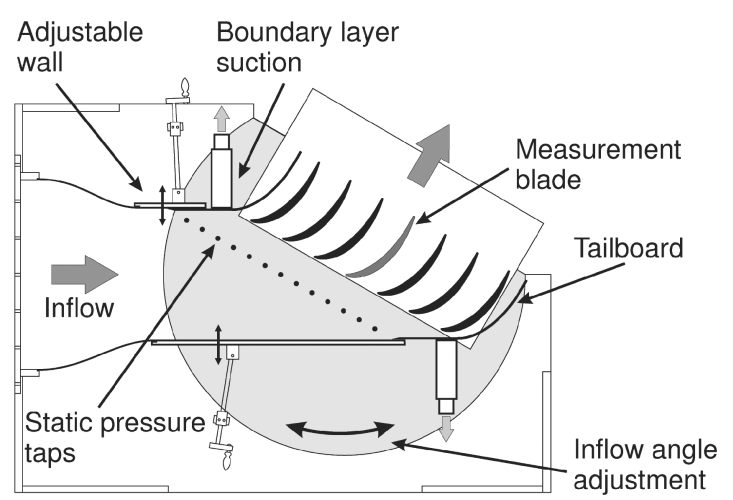

Fig. 3 Cascade test rig at open wind tunnel

(b)

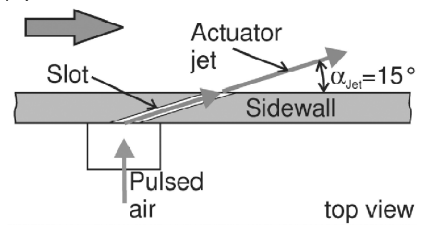

(c)

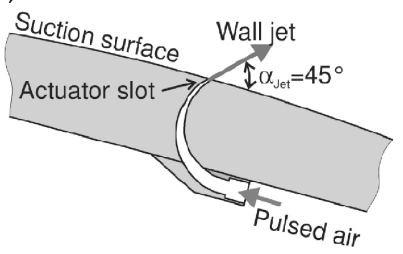

Fig. 4 (a) Stator blade with actuator setup; (b) sidewall actuator (cross-section in top-view); and (c) blade actuator (cross-section) 
solenoid valves with a switching frequency up to $250 \mathrm{~Hz}$ are used for pulsing the compressed air in both actuator concepts. Hereby, the frequency and the amplitude are varied and the actuator duty cycle is held constant at 50 per cent.

\subsection{Numerics}

The use of CFD by means of Reynolds-averaged Navier-Stokes (RANS) simulations has become a helpful tool within the industrial design process of turbomachinery blades. Due to the cooperation with Rolls-Royce Deutschland, an industrial numerical approach is pursued, employing a commercial software package. Since URANS methods do not comprise all details of the complex flow phenomena involved in AFC via jets, the interest lies in the feasibility of correctly predicting the impact of AFC.

\subsubsection{Flow solver}

For the numerical simulations has been employed the software package $F I N E^{T M}$ / Turbo v.8.6-1 from NUMECA International [14]. Since the appendant grid generator for turbomachinery (AutoGrid) offers only limited meshing opportunities for linear cascades, grids are created with G3DMESH, a tool developed at DLR Cologne by Weber [15]. Grid preprocessing as well as local refinements for actuator nozzle attachments are realized within IGG, a structured grid generator from NUMECA International.

The flow solver EURANUS solves the 3D RANS equations on general structured non-orthogonal multi-block grids. The incorporated numerical algorithm is an explicit four stage Runge-Kutta scheme [16]. Various convergence acceleration techniques are employed, such as implicit residual smoothing, dual time stepping, and a full multigrid algorithm. Space integration is performed using a second order cell-centred finite volume discretization with fourthorder artificial dissipation.

\subsubsection{Boundary conditions}

The one-equation turbulence model of Spalart and Allmaras [17] is used in combination with fixed transition on the blade surfaces. Transition is realized by taking into account the influence of an intermittency parameter which triggers the production term of the turbulence model. This intermittency parameter is zero within the laminar and one within the turbulent flow regime. Transition position is evaluated against an oil-flow visualization from the experiment. Best concurrence is achieved using a step function at

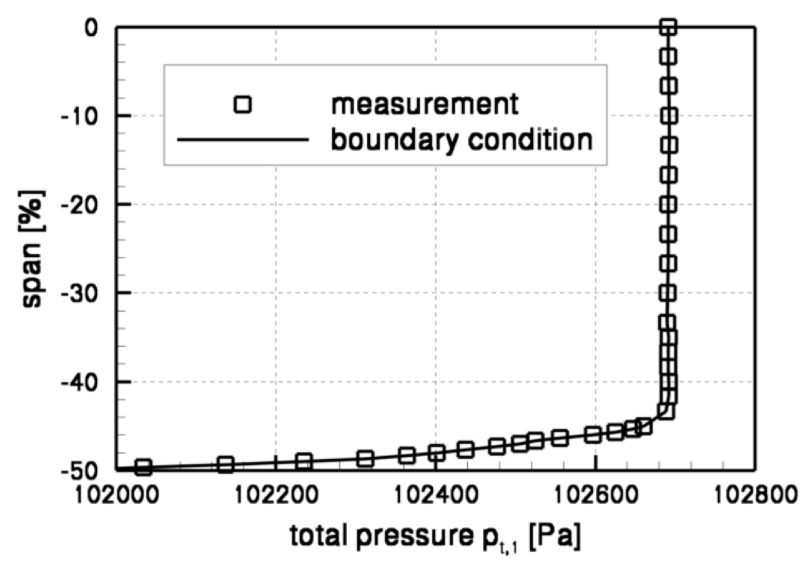

Fig. 5 Total pressure profile

Table 2 Numerical boundary conditions

\begin{tabular}{llll}
\hline Inlet & & & \\
Inflow angle & $\beta_{1}$ & 60 & $\mathrm{deg}$ \\
Total temperature & $T_{t, 1}$ & 293.95 & $\mathrm{~K}$ \\
Total pressure & $p_{t, 1}(z)$ & cf. Fig. 5 & $\mathrm{~Pa}$ \\
Turb. viscosity & $v_{t, 1}$ & $7.154 \cdot 10^{-5}$ & $\mathrm{~m}^{2} / \mathrm{s}$ \\
Outlet & $p_{2}$ & 102417 & $\mathrm{~Pa}$ \\
Static pressure & & & \\
\hline
\end{tabular}

23 per cent suction side and 3 per cent pressure side length, respectively.

In order to account for the incoming boundary layer, a spanwise profile of the total pressure $p_{t, 1}(z)$ is imposed, which is measured at a plane corresponding to the inlet of the computational domain, cf. Fig. 5. Due to symmetry reasons, the profile is shown over the half span only. The turbulent viscosity at the inlet $v_{t, 1}$ is chosen in such a way that the inlet turbulence intensity is in accordance with the measurement. The desired inlet flow Mach number $M a_{1}=0.1$ is achieved by variation of the constant static pressure $p_{2}$ imposed at the outlet boundary. The boundary conditions for all calculations are listed in Table 2.

\subsubsection{Computational domain}

The meshes used for the simulations are blockstructured and approximately 20 grid lines resolve the boundary layer down to the viscous sublayer. All meshes generated provide three full multigrid levels. A previously performed mesh study [18] showed that the use of 2.0 million grid cells ensures sufficient resolution of the base flow. The used mesh resolves the span with 97 points and the maximum dimensionless wall distance is $y_{\max }^{+}=2.3$. An overview of the mesh is depicted in Fig. 6(a). 

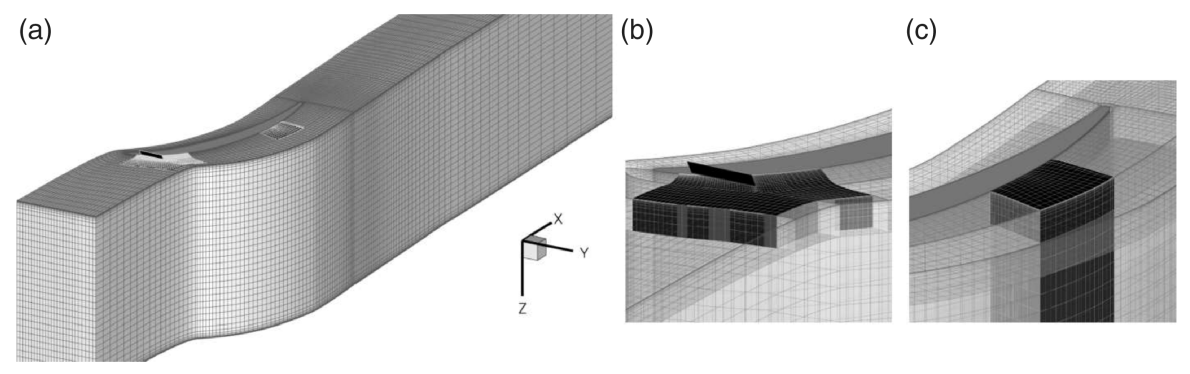

Fig. 6 (a) Overview of the computational domain; (b) refined area at the casing end wall; and (c) refined area at the suction side - only every second grid cell is shown

The actuation is realized via nozzle blocks which spatially resolve the actuators at the according walls. A neat momentum transfer is guaranteed by resolving the actuator slot within the main computational domain. Therefore, the grid is refined locally at the vicinity of the actuation as shown in detail in Fig. 6(b) for the casing end wall and Fig. 6(c) for the blade suction side, respectively. The number of grid cells increases to 2.5 million. Thus, an acceptable mean of spatial resolution and computational effort is granted, as presented in previous studies [18].

\subsection{Data evaluation}

Data evaluation is conducted by means of different parameters and coefficients, calculated from the experimental and numerical results. Thus, the passage flow field can be characterized, the actuation depending on the passage flow described, and the efficiency of the cascade without and with actuation determined. For all data analyses, the evaluation planes are located one-third of the chord length upstream of the leading edge and one-third downstream of the trailing edge.

\subsubsection{Passage flow}

The pressure coefficient $c_{p}$ is defined as the ratio of the difference of the local pressure $p_{x}$ and the inlet pressure $p_{1}$ to the dynamic pressure at the inlet $q_{1}$ : $c_{p}=\left(p_{x}-p_{1}\right) / q_{1}$. With the help of the $c_{p}$ distribution at midspan of the blade, the local passage flow along the suction and pressure side is comparable between experimental measurements and numerical simulations.

In previous works, the global performance of the stator cascade and the impact of the flow control were determined by the mass-averaged total pressure loss and the area-averaged static pressure rise.
The total pressure loss $\zeta_{q_{1}}$ and the pressure rise $\Delta p / q_{1}$ are defined in equations (1) and (2)

$$
\begin{gathered}
\zeta_{q_{1}}=\frac{p_{t, 1}-p_{t, 2}}{q_{1}} \\
\frac{\Delta p}{q_{1}}=\frac{p_{2}-p_{1}}{q_{1}}
\end{gathered}
$$

\subsubsection{Actuator}

The momentum flux of the actuator is quantified by $c_{\mu}$ (equation (3)) which represents the momentum of the jet in relation to the momentum of the incoming flow. Here, the effective value of the jet velocity is considered, which is mathematically represented by the RMS-value $V_{\text {jet,RMS. Additionally, the injected }}$ mass flow $\dot{m}_{\text {jet }}$, the dynamic pressure at the cascade inlet $q_{1}$ and the area of the cascade inlet plane $A_{1}$ are used for its calculation

$$
c_{\mu, \mathrm{RMS}}=\frac{\dot{m}_{\mathrm{jet}} \cdot V_{\mathrm{jet}, \mathrm{RMS}}}{q_{1} \cdot A_{1}}
$$

For unsteady excitation, the actuator frequency is normalized by the quotient of a geometrical reference length and a reference velocity. Here, the blade chord $l$ and the inflow velocity $V_{1}$ are used to calculate the reduced frequency $F^{+}=f \cdot l / V_{1}$.

\subsubsection{Efficiency}

The main goal using AFC in a compressor cascade is to raise the global efficiency of the stator. One approach of calculating the efficiency for diffusers is given by Bräunling [19] which can be adapted to the cascade passage for the base flow case. The efficiency relates the benefit to the costs. In a compressor stator, the kinetic energy at the passage outlet $P_{2}=V_{2}^{2} / 2$ and the specific volumetric work across the passage $w=\int_{1}^{2} v \cdot \mathrm{d} p$ can be seen as benefit. The kinetic energy of the inlet flow $P_{1}=V_{1}^{2} / 2$ represents the costs. 
(a)

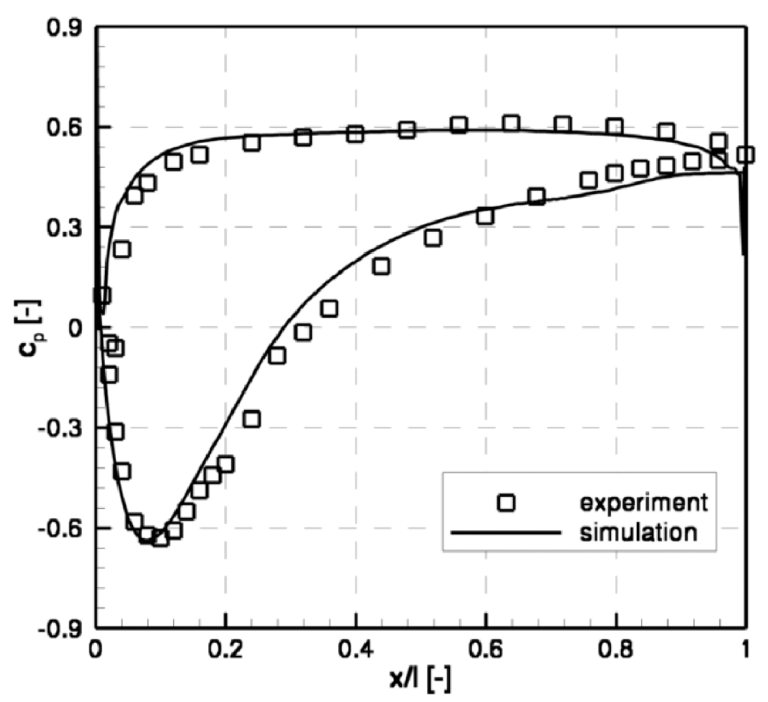

(b) $x / I=50 \%$
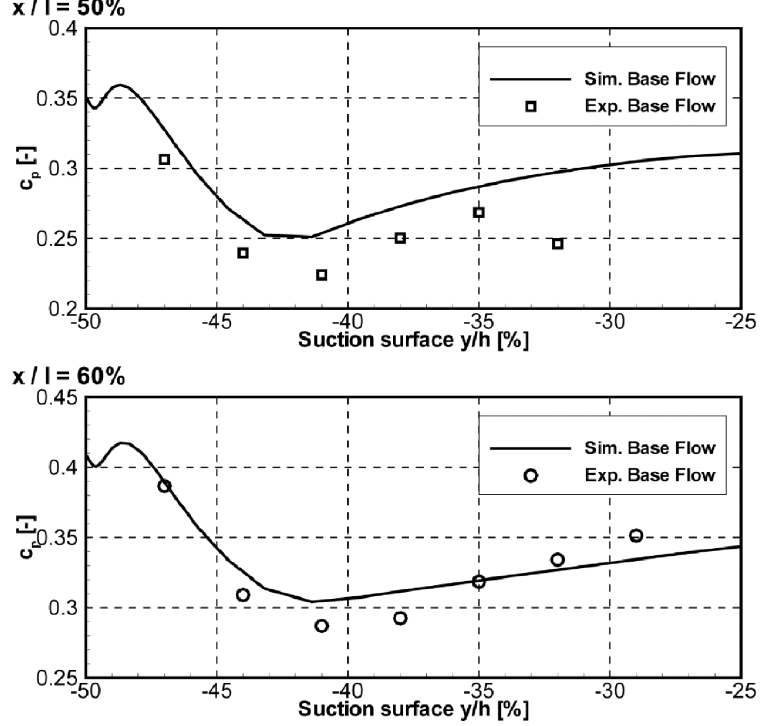

Fig. 7 Experimental and numerical results of the base flow case: (a) $c_{p}$-distribution at midspan and (b) $c_{p}$-distribution at $x / l=50 \%$ and $60 \%$ in spanwise direction

Using AFC, the pressure rise across the passage is increased. But, for the efficiency calculation, the cost of the actuation has to be taken into account. The above-described efficiency of a diffuser is adapted to the actively controlled compressor stator cascade. The kinetic energy of the actuator jet $P_{\text {jet }}=V_{\text {jet }}^{2} / 2$ has to be considered as additional cost, while the specific volumetric work of the injected mass $w_{\text {jet }}=\int_{\text {jet }}^{2} v \cdot \mathrm{d} p$ can be seen as benefit. In order to correctly take the individual impact into account, all parameters are massflow-weighted and the efficiency of a forced cascade flow is calculated as follows

$$
\begin{aligned}
\eta_{\mathrm{AFC}} & =\frac{\text { benefit }}{\text { costs }}=\frac{P_{2} \cdot \dot{m}_{2}+w \cdot \dot{m}_{1}+w_{\mathrm{jet}} \cdot \dot{m}_{\mathrm{jet}}}{P_{1} \cdot \dot{m}_{1}+P_{\mathrm{jet}} \cdot \dot{m}_{\mathrm{jet}}} \\
& =\frac{V_{2}^{2} / 2 \cdot \dot{m}_{2}+w \cdot \dot{m}_{1}+w_{\mathrm{jet}} \cdot \dot{m}_{\mathrm{jet}}}{V_{1}^{2} / 2 \cdot \dot{m}_{1}+V_{\mathrm{jet}}^{2} / 2 \cdot \dot{m}_{\mathrm{jet}}}
\end{aligned}
$$

\section{RESULTS}

\subsection{Base flow}

The 3D flow structures evolving in the passage have been thoroughly discussed in previous publications. In order to give an impression of the numerical capabilities to capture these complex phenomena, the pressure distribution at midspan is compared to experimental data in Fig. 7(a). The suction peak at 10 per cent chord is well matched. Even though transition is considered within the simulation and a laminar separation bubble is calculated, the pressure plateau observed within the experiment is missing in the numerical data. Between 30 per cent and 60 per cent chord, the pressure is slightly overestimated by the numerics. This is related to a distinct discrepancy between the experiment and the simulation in the area where the main flow intersects the secondary flow from the end walls [12]. The 3D separation line is shaped concave in the simulation and convex in the experiment. Greater discrepancies can be found at around 80 per cent chord because of the flow separation occurring slightly downstream in the simulation.

The footprint of the secondary flow structure, here namely the corner vortex, can be clearly observed in the spanwise pressure distributions at 50 per cent and 60 per cent chord, shown in Fig. 7(b). The downstream expansion of the vortex is distinctly illustrated. The simulation slightly overestimates the pressure minimum and underpredicts the gradient in the direction of the blade centre.

The passage flow characteristics are described by the integral values of total pressure loss and pressure rise, and the efficiency of the cascade. In Table 3 , the values of the base flow are compared between experiment and simulation. In spite of the above-described discrepancies within the complex flow field, the global values differ by less than 2 per cent.

Evaluation of the base flow shows a very complex 3D flow topology evolving in the passage. Since turbulence models are to some extent inevitably empirical, even state-of-the-art approaches struggle with the complexity of turbulence closure arising from the strongly separated boundary layers. The applied linear eddy viscosity model can only capture the first-order effects of the Reynolds stress 
anisotropy and streamline curvatures which are strongly pronounced in secondary flow fields. The Spalart-Allmaras turbulence model therefore tends to overestimate the secondary flow regime which was also found by Lewin et al. [20]. Despite these limitations, the base flow is well predicted by the simulation.

\subsection{Sidewall actuation}

The effect of the sidewall actuation on the secondary flow is exemplarily illustrated for a pulsating jet with a momentum coefficient of $c_{\mu, \mathrm{RMS}}=0.85$ per cent and a reduced frequency of $F^{+}=1.07$. Figure 8 displays the time-averaged velocity field of the corner vortex from the experiment and the simulation. For

Table 3 Cascade performance data

\begin{tabular}{llll}
\hline Parameter & & Experiment & Simulation \\
\hline Total pressure loss & $\zeta_{q_{1}}$ & 0.106 & 0.107 \\
Static pressure rise & $\Delta p / q_{1}$ & 0.565 & 0.554 \\
Stator efficiency & $\eta_{\text {Base }}$ & 0.825 & 0.832 \\
\hline
\end{tabular}
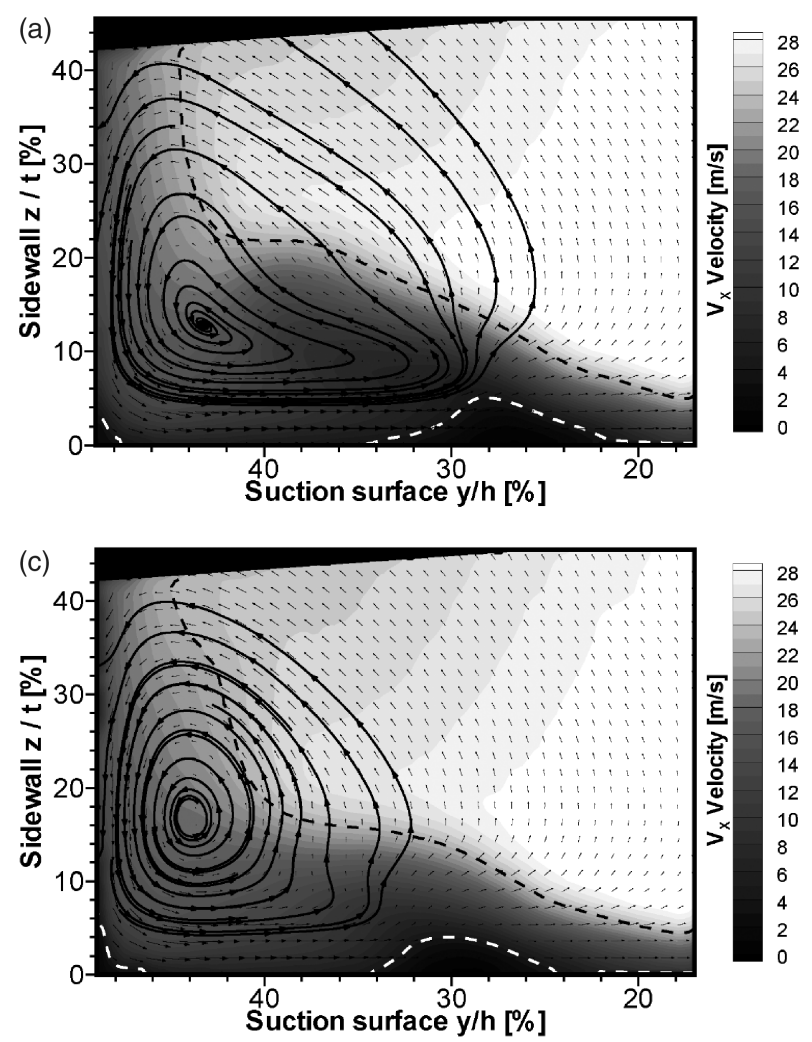

comparative reasons, the base flow and the forced flow case are shown. The corner vortex, driven by a pitchwise pressure gradient across the passage, is highlighted by streamlines of the in-plane components. Iso-lines for $V_{x}=4 \mathrm{~m} / \mathrm{s}$ (white) and $23 \mathrm{~m} / \mathrm{s}$ (black) enclose the regions of low velocity with local areas of recirculation on the blade surface $\left(V_{x}<4 \mathrm{~m} / \mathrm{s}\right)$ and the undisturbed passage flow $\left(V_{x}>23 \mathrm{~m} / \mathrm{s}\right)$.

The experimental and numerical results of the base flow case (Figs 8(a) and (b)) show a similar vortex core position and the region of the undisturbed passage flow is well estimated by the simulation, though slight differences can be found for the vortex formation, illustrated by the streamlines. The region of the recirculation, indicated by the low velocity on the blade surface $\left(V_{x}<4 \mathrm{~m} / \mathrm{s}\right)$, is overestimated in size by the simulation. Since this region is dominated by high shear between the main passage flow and the corner vortex, high local velocity and pressure fluctuations occur which have to be challenged by the numerical code. Still, the position of recirculation is in good agreement between the experiment and the simulation.
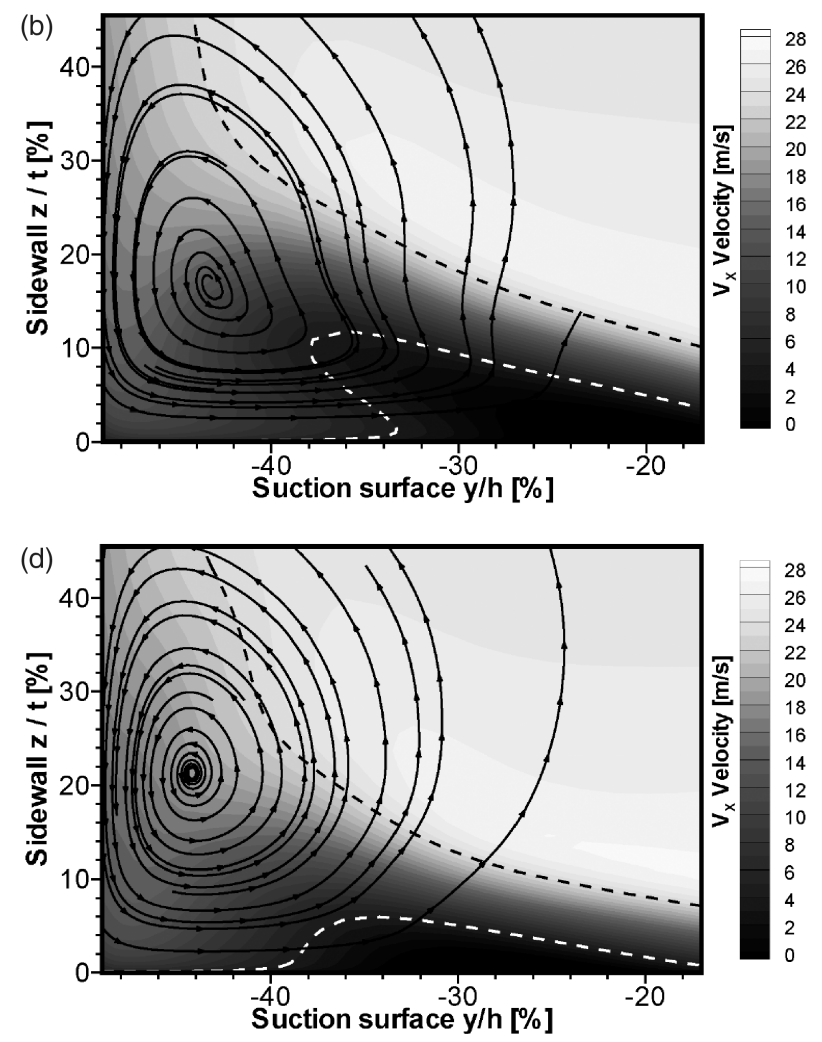

Fig. 8 Comparison of experimental ((a) and (c)) and numerical ((b) and (d)) velocity fields of the corner vortex on a slice perpendicular to the blade suction side at $x / l=60 \%$ for the base flow and the forced flow case with $c_{\mu, \mathrm{RMS}}=0.85 \%$ and $F^{+}=1.07$ : (a) base flow: time-averaged 3CPIV, (b) base flow: steady state numerical data, (c) forced flow: time-averaged 3C-PIV and (d) forced flow: time-averaged numerical data 

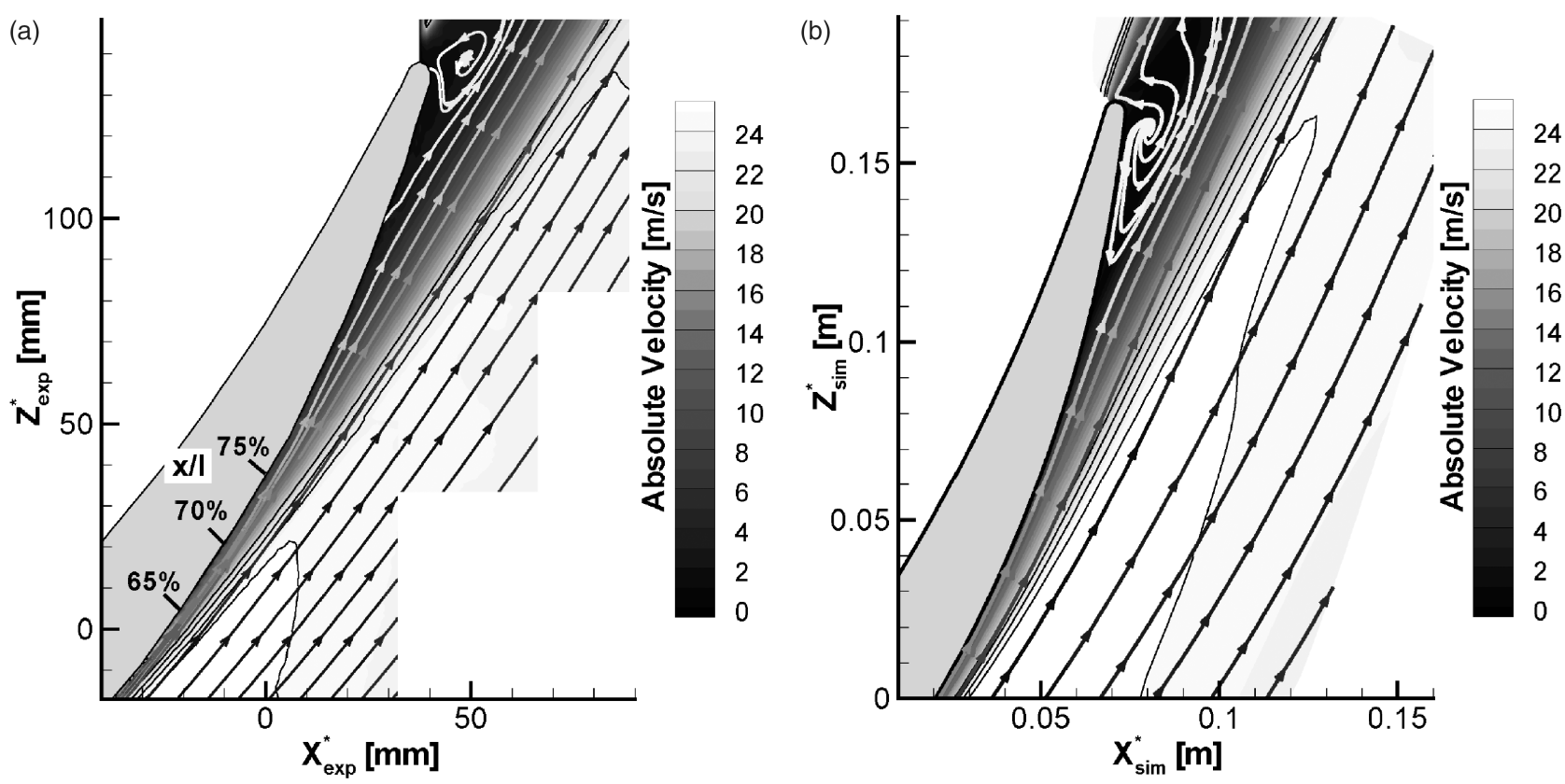

Fig. 9 Comparison of experimental and numerical velocity field data for the base flow case: (a) experimental and (b) numerical data

AFC out of the sidewalls shifts the corner vortex toward the casing. This trend can be clearly seen in Fig. 8(c) for the experiment and in Fig. 8(d) for the simulation. The undisturbed passage flow $\left(V_{x}>23 \mathrm{~m} / \mathrm{s}\right)$ is enlarged and thus the passage blockage reduced. The area of recirculation $\left(V_{x}<4 \mathrm{~m} / \mathrm{s}\right)$ also changes its position towards the sidewall. In the experiment, the region maintains its size with respect to the base flow. In the simulation the size is still overestimated and the formation differs compared to the experimental results. Compared to the numerical result of the base flow, a changed formation of the corner vortex results in a reduced size of the area enclosed by the iso-line $V_{x}=4 \mathrm{~m} / \mathrm{s}$. All in all, the experimental flow field of the corner vortex is adequately captured by the numerical results. Same trends are shown and a similar response of the secondary flow field on the AFC device is observed.

\subsection{Blade actuation}

The pressure-induced flow separation is suppressed with the blade actuators which are aligned upstream close to the separation line on the blade's suction surface. Figure 9 shows the time-averaged velocity fields of the base flow at midspan out of the experiment and the simulation. The position of the flow separation can be observed between 70 per cent and 75 per cent chord length, with the tendency of a slightly delayed separation in the simulation.
The jet forces the separated boundary layer to reattach to the contour of the blade. Due to the pulsed wall jet, high momentum fluid is transferred close to the blade surface. The separation line is shifted towards the trailing edge and the deflection enlarges. The forcing mechanisms for two reduced frequencies $\left(F^{+}=0.43\right.$ and 1.28$)$ are shown by means of velocity fields in Fig. 10 for pulsed jets with a momentum coefficient of $c_{\mu, \mathrm{RMS}}=1.5$ per cent.

The velocity fields are phase-averaged based on the actuator cycle and are displayed for four actuator phase angles $(\phi)$. The blowing cycle starts with $\phi=0^{\circ}$ and has a duty cycle of 50 per cent. Using the lower forcing frequency of $F^{+}=0.43$, the separation is suppressed periodically with the blowing phase. The flow attaches to the blade surface $\left(\phi=90-180^{\circ}\right)$ and starts to separate during the actuator's off-cycle $\left(\phi=270^{\circ}\right)$. With the initial jet of the next actuator cycle, the flow is completely separated. This interaction can be described by a periodic excitation which is below the minimum forcing frequency needed to suppress the flow separation completely.

The global forcing mechanisms and trends are in good agreement between the numerical and experimental results. Only details in the flow field differ, like the vortex pair of the initial actuator jet. In addition, the size of the separated flow is overestimated in the simulation at this phase angle $\left(\phi=0^{\circ}\right)$. The detachment process starts faster when the flow has attached to the blade surface, which results in a slight phase shift between the experiment and the numerics. 

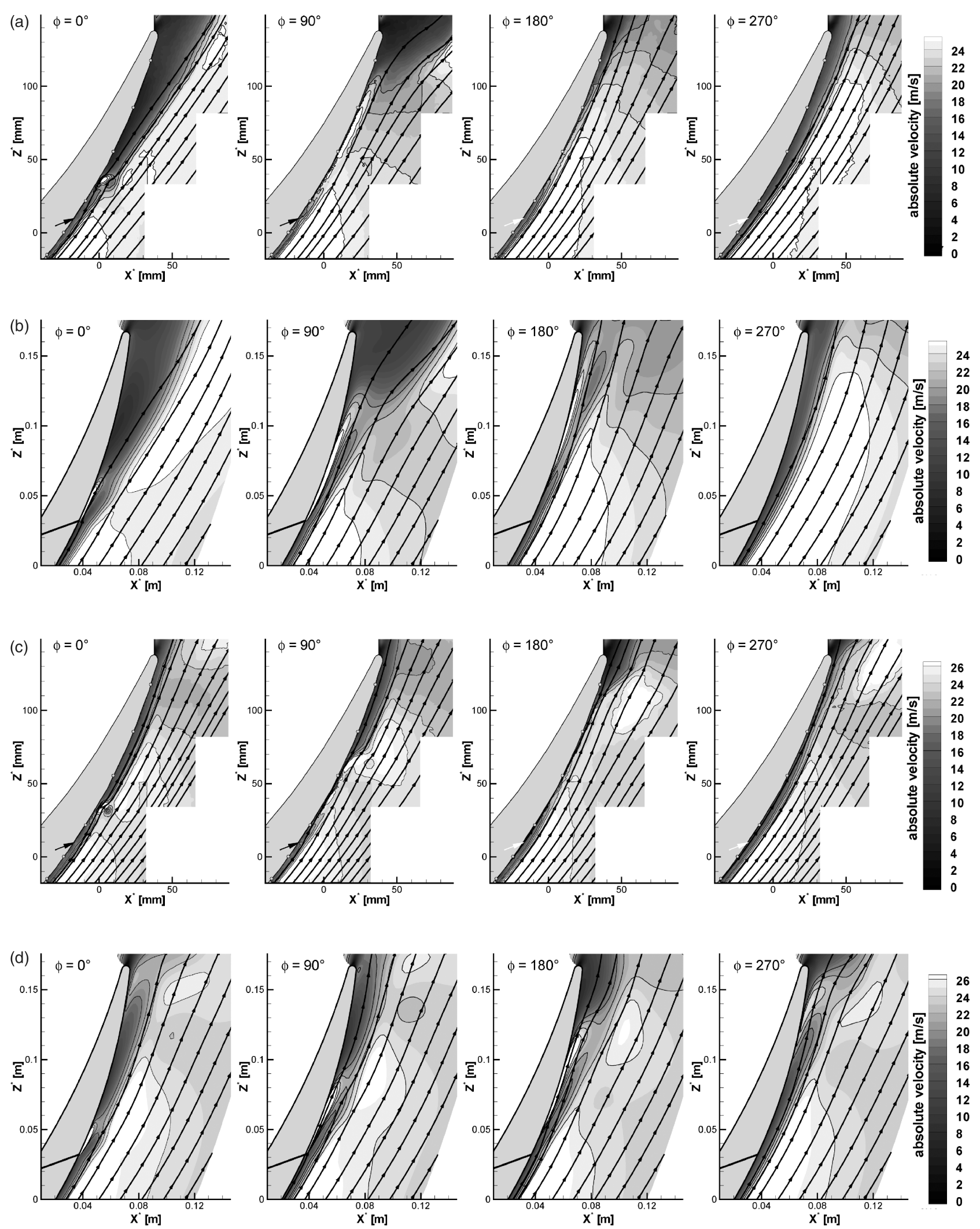

Fig. 10 Experimental ((a) and (c)) and numerical ((b) and (d)) velocity fields of the pressureinduced flow separation on a slice at midspan for different forcing frequencies: (a) forced flow with $c_{\mu, \mathrm{RMS}}=1.5 \%$ and $\mathrm{F}^{+}=0.43(40 \mathrm{~Hz})$ : phase-averaged PIV data, (b) forced flow with $c_{\mu, \mathrm{RMS}}=1.5 \%$ and $\mathrm{F}^{+}=0.43(40 \mathrm{~Hz})$ : phase-averaged numerical data, (c) forced flow with $c_{\mu, \mathrm{RMS}}=1.5 \%$ and $\mathrm{F}^{+}=1.28(120 \mathrm{~Hz})$ : phase-averaged PIV data and (d) forced flow with $c_{\mu, \mathrm{RMS}}=1.5 \%$ and $\mathrm{F}^{+}=1.28(120 \mathrm{~Hz})$ : phase-averaged numerical data 

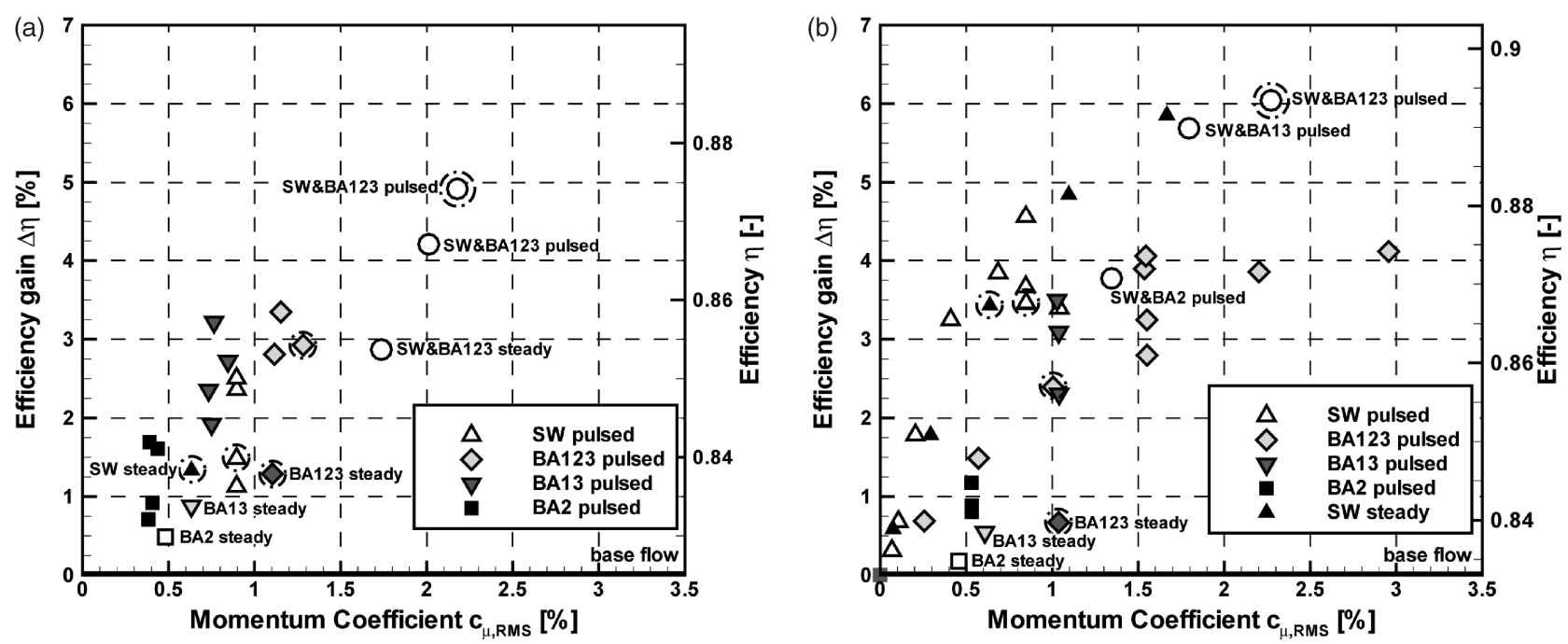

Fig. 11 Comparison of numerical and experimental efficiency values: (a) experimental and (b) numerical efficiency

Using an increased forcing frequency of $F^{+}=1.28$, the separation is completely suppressed. The next blowing cycle starts before the flow detaches from the blade surface. Thus, the separated shear layer has a certain receptivity to this forcing frequency. Comparing the simulation to the experiment, the separated region is still overestimated. The jet structures close to the blade surface differ in both methods. In the simulation, the actuator jet remains much closer to the wall. The interaction of the wall jet with the separated shear layer shows some slight differences, but again the global trends are in very good agreement.

\subsection{Efficiency}

In order to rate the benefit of the different flow control concepts, the efficiency of the controlled cascade is plotted against the momentum coefficient of the actuation. Representative experimental and numerical data are shown in Fig. 11 for both control concepts applied separately and in combination considering steady and pulsed blowing. The investigated parameter from experiment and simulation may differ. Several points for one concept at the same momentum coefficient indicate a frequency variation. The difference in base flow efficiency between experiment and numerics is compensated by regarding the efficiency gain $\Delta \eta$. Thus, the base flow efficiency is zero for both experimental and numerical data on the left ordinate. The positive effect of both concepts is equally captured within the measurements and the numerics. All parameters investigated for both concepts result in an increase of the cascade performance. Partially, different setups are considered explaining some quantitative discrepancies between experiment and simulation. All in all, the global outcome is very well captured within the simulations and similar trends can be found as observed experimentally. For comparative setups, the quantitative outcome of experiment and simulation differs by approximately 1 per cent in efficiency increase. The numerics tend to slightly overpredict the benefit of the sidewall actuator and underestimate the impact of the blade actuation.

The datasets show that for both concepts investigated, steady blowing is less efficient than pulsed blowing, especially for small momentum coefficients, the pulsed jets perform better. Furthermore, both concepts are equally able to increase the compressor performance. For the blade actuators, the use of the middle segment BA2 turns out to be inefficient since actuating with only the two outer segments BA1 +3 results in the same efficiency gain as using all three segments. The best results are achieved by running the two concepts in combination, as can be seen in the experimental and numerical results.

The data considered represent actuation with blowing ratios of $0.7 \leqslant V_{\text {jet,RMS }} / V_{1} \leqslant 3.5$ for various excitation frequencies. Due to the small areas of the actuators, even for the highest blowing ratios investigated and combined actuation at sidewalls and blade, the mass flowrates of the pulsed jets are below 0.5 per cent of the passage mass flow. Steady jets come along with higher mass flowrates. Thus, another important benefit of the pulsed blowing is observed.

For a more detailed comparison between experiment and numerics, five setups using similar 
Table 4 Efficiency of different control concepts

\begin{tabular}{|c|c|c|c|c|c|c|c|c|c|c|c|c|}
\hline \multirow{2}{*}{$\begin{array}{l}\text { Setup } \\
\text { Parameter }\end{array}$} & \multicolumn{6}{|c|}{ Experiment } & \multicolumn{6}{|c|}{ Simulation } \\
\hline & \multicolumn{2}{|c|}{$F^{+}$} & \multicolumn{2}{|c|}{$V_{\text {jet }} / V_{1}$} & $\eta$ & $\Delta \eta(\%)$ & \multicolumn{2}{|c|}{$F^{+}$} & \multicolumn{2}{|c|}{$V_{\text {jet }} / V_{1}$} & $\eta$ & $\Delta \eta(\%)$ \\
\hline SW steady & \multicolumn{2}{|c|}{-} & \multicolumn{2}{|c|}{2.0} & 0.838 & 1.3 & \multicolumn{2}{|c|}{ - } & \multicolumn{2}{|c|}{2.0} & 0.867 & 3.4 \\
\hline BA123 steady & \multicolumn{2}{|c|}{-} & \multicolumn{2}{|c|}{1.4} & 0.838 & 1.3 & \multicolumn{2}{|c|}{-} & \multicolumn{2}{|c|}{1.3} & 0.839 & 0.6 \\
\hline SW pulsed & \multicolumn{2}{|c|}{1.28} & \multicolumn{2}{|c|}{2.9} & 0.840 & 1.5 & \multicolumn{2}{|c|}{1.28} & \multicolumn{2}{|c|}{2.9} & 0.867 & 3.4 \\
\hline BA123 pulsed & \multicolumn{2}{|c|}{1.28} & \multicolumn{2}{|c|}{1.8} & 0.854 & 2.9 & \multicolumn{2}{|c|}{1.07} & \multicolumn{2}{|c|}{1.6} & 0.856 & 2.3 \\
\hline SW\&BA123 & SW & BA & SW & BA & & & SW & BA & SW & BA & & \\
\hline pulsed & 1.61 & 1.28 & 2.92 & 1.8 & 0.875 & 5.0 & 1.28 & 1.28 & 2.9 & 2.0 & 0.893 & 6.0 \\
\hline
\end{tabular}

actuation parameters are compared in Table 4. These setups are also highlighted by circles in the efficiency plot (cf. Fig. 11).

\section{CONCLUSIONS}

Experimental and numerical investigations of AFC are performed on a highly loaded compressor cascade. A complex and highly 3D passage flow field occurs due to the low aspect ratio and overcritical turning of the stator blade. Two actuator concepts are used for suppressing the flow separation and reducing the secondary flow structures. By blowing out of the sidewalls, the corner vortices are repositioned towards the casing and thus the passage blockage is reduced. The flow separation in the rear part of the blade is suppressed by means of blowing out of slots in the blade suction surface. With both AFC concepts, the turning of the blade can be increased and the blockage of the passage flow is reduced, resulting in an increased static pressure rise and decreased total pressure loss.

Comparisons between URANS simulations and experiments performed on the compressor cascade are presented. Therefore, detailed flow field data involving the physics of the flow control devices were extracted from the numerical results, covering the experimental PIV and pressure probe measurements. For the base flow, local values like the pressure distribution at midspan and in the region of the corner vortices show some local discrepancies. The passage flow characteristics, like the total pressure loss, the pressure rise and the efficiency are in excellent agreement with differences less than 2 per cent. Comparing the local flow fields of the corner vortex and its response to the actuator cycle, the same good trends can be estimated and the effects on the corner vortex were comparable. The region of the secondary flow is characterized by high fluctuations and shear stresses in the flow field, which represent a challenge for the modelling involved in URANS simulation. Comparing the interaction mechanism of the blade actuator jet, the global trends can be numerically covered. The response of the separated shear layer to the wall jet pulsing at two different forcing frequencies matches adequately well between experiment and simulation. Only a slight phase shift and local differences of the vortex formation close to the actuator jet occur. Basically, the URANS computations predict the same trend as found by the experiments without resolving the unsteady jets temporally or spatially in detail. Since the entire flow field has a very steady character, no dominant frequency is found that can be used as a forcing frequency. The passage flow is therefore excited by a forcing frequency which may improve numerical flow prediction, since the frequencies of the small scale turbulent structures are not resolved within a RANS approach. Discrepancies between experiment and simulation may also arise from the ideal modelling of a dirac pulse in the numerics compared to a real pulsed jet produced by a fast-switching valve.

The efficiency of the stator cascade is used as the global parameter for rating the benefit of the AFC concepts. All in all, the global outcome is very well captured within the simulations and similar trends can be found as observed experimentally. The quantitative outcome of experiment and simulation differs by approximately 1 per cent in efficiency increase. The numerics tend to slightly overpredict the benefit of the sidewall actuator and underestimate the impact of the blade actuation. For similar actuation parameter of the pulsed excitation, a maximal efficiency gain of 5 per cent can be reached in the experiment and 6 per cent in the simulation. Using steady-state blowing, the efficiency is significantly reduced and comes along with higher mass flowrates. The jet velocity ratio varies between $0.7 \leqslant V_{\text {jet,RMS }} /$ $V_{1} \leqslant 3.5$ and a mass flow ratio below 0.5 per cent of the passage mass flow is used for all investigations.

\section{FUNDING}

This work was supported by the DFG (German Research Foundation) [project number TFB/SFB557]. 


\section{ACKNOWLEDGEMENTS}

The results presented were achieved in cooperation with Rolls-Royce Deutschland Ltd \& Co. KG as part of the Collaborative Research Center 557, Control of turbulent shear flows, at TU Berlin.

\section{(C) Authors 2011}

\section{REFERENCES}

1 Lord, W. K., MacMartin, D. G., and Tillman, G. Flow control opportunities in gas turbine engines. AIAA paper 2000-2234, 2000.

2 Wennerstrom, A. J. Highly loaded axial flow compressors: history and current developments. ASME J. Turbomach., 1990, 112, 567-578.

3 Bright, M., Culley, D., Braunscheidel, E., and Welch, G. Closed loop active flow separation detection and control in a multistage compressor. Technical Report NASA TM-2005-213553, NASA, 2005.

4 Hergt, A., Meyer, R., and Engel, K. Experimental investigation of flow control in compressor cascades. ASME paper GT2006-90415, 2006.

5 Nerger, D. Aktive Strömungsbeeinflussung in ebenen Statorgittern hoher aerodynamischer Belastung durch Ausblasen. PhD Thesis, Technische Universität Braunschweig, 2009.

6 Greenblatt, D. and Wygnanski, I. J. The control of flow separation by periodic excitation. Prog. Aerosp. Sci., 2000, 36, 487-545.

7 Langston, L. S. Crossflows in a turbine cascade passage. J. Eng. Power (ASME), 1980, 102, 866-874.

8 Hecklau, M., van Rennings, R., Zander, V., Nitsche, W., Huppertz, A., and Swoboda, M. Particle image velocimetry of active flow control on a compressor cascade. Exp. Fluids, 2011, 50(4), 799-811.

9 Hecklau, M., Wiederhold, O., Zander, V., King, R., Nitsche, W., Huppertz, A., and Swoboda, M. Active separation control with pulsed jets in a critically loaded compressor cascade. AIAA J., 2011, 49(8), 1729-1739.

10 Hecklau, M., Zander, V., Peltzer, I., Nitsche, W., Huppertz, A., and Swoboda, M. Notes on numerical fluid mechanics and multidisciplinary design. In Active flow control II - NNFM (Ed. R. King), 2010, (Springer, Berlin), pp. 171-186. DOI: 10.1007/978-3642-11735-0_12.

11 Gmelin, C., Steger, M., Wassen, E., and Thiele, F. Notes on numerical fluid mechanics and multidisciplinary design. In Active flow control II - NNFM (Ed. R. King), 2010, (Springer, Berlin), pp. 203-219. DOI: 10.1007/978-3-642-11735-0_14.

12 Gmelin, C., Steger, M., Wassen, E., Thiele, F., Huppertz, A., and Swoboda, M. Unsteady RANS simulations on flow control in a compressor cascade using pulsed jets at the blade. AIAA paper 2010-4588, 2010.

13 Mertens, D., Thiele, F., Swoboda, M., and Huppertz, A. Transition modeling effects on the simulation of a stator cascade with active flow control. ASME paper GT2008-50697, 2008.

14 NUMECA International, FINE $E^{T M} /$ Turbo v8, user manual. NUMECA Int., Brussels, 2007.

15 Weber, A. G3DMESH v4.5.4. Institut für Antriebstechnik, Köln, DLR, 2008.

16 Jameson, A. and Baker, T. J. Multigrid solution of the euler equations for aircraft configurations. AIAA paper 1984-93, 1984.

17 Spalart, P. R. and Allmaras, S. R. A one-equation turbulence model for aerodynamic flows. AIAA paper 1992-439, 1992.

18 Gmelin, C., Steger, M., Zander, V., Nitsche, W., Thiele, F., Huppertz, A., and Swoboda, M. Numerical investigations of active flow control using synthetic jets on a highly loaded compressor stator cascade. ASME paper FEDSM-ICNMM201030725, 2010.

19 Bräunling, W. J. G. Flugzeugtriebwerke, 2009 (Springer, Heidelberg).

20 Lewin, E., Kozulovic, D., and Stark, U. Experimental and numerical analysis of hub-corner stall in compressor cascades. ASME paper GT2010-22704, 2010.

\section{APPENDIX}

\section{Notation}

$c_{p} \quad$ pressure coefficient

$p, p_{t}, q \quad$ static, total, dynamic pressure

$w \quad$ specific volumetric work

$x, y, z \quad$ coordinate system

$\mathrm{P} \quad$ specific kinetic energy

V velocity

$\zeta_{q_{1}} \quad$ total pressure loss

$\rho$ density

$\eta \quad$ efficiency

\section{Super-and subscripts}

$1 \quad$ inlet

2 outlet

* transformed coordinates

afc forced flow

base base flow

exp experiment

jet actuator jet, position

sim simulation 